\title{
Tabique vaginal transverso
}

\author{
Alberto Duarte Contreras*
}

\begin{abstract}
RESUMEN: El septo vaginal transverso se diagnostica en la recién nacida o durante la pubertad temprana. En la recién nacida las secreciones se colectan en la vagina y forman el hidrocolpos. Durante la pubertad temprana la sangre menstrual recolectada forma el hematocolpos. Se presentan dos casos: una recién nacida con masa abdominal por hidrocolpos y otra en pubertad temprana con masa abdominal por hematocolpos. Se discuten los casos.
\end{abstract}

PALABRAS CLAVES: Septo vaginal transverso, hidrocolpos, hematocolpos, anomalía vaginal.

SUMMARY: The transverse vaginal septum is diagnosed in new borne or during early puberty. In the neonate genital tract secretions may collect in the vagina and form the hydrocolpos. In the early puberty the menstruation may collect and form the hematocolpos. The findings included two cases: a new born with hydrocolpos and another with hematocolpos and early puberty. A discussion of these two cases.

KEY WORDS: Transverse vaginal septum, hydrocolpos, hematocolpos, vaginal anomaly.

\section{Introducción}

El tabique vaginal transverso es la malformación congénita genital que se encuentra con mayor frecuencia: constituye un poco más del $10 \%$ del total de anormalidades congénitas del tracto genital femenino (1). Su presencia se debe investigar en la recién nacida a término que no presente secreción mucosa vaginal en los primeros días de vida, lo mismo que en todas las recién nacidas prematuras, mediante la prueba de permeabilidad del canal vaginal, durante el examen general rutinario que practique el pediatra o el médico que atienda el parto. El diagnóstico es más fácil en la recién nacida a término que en la prematura (2).

La presencia del tabique pasa desapercibida para la mayoría de los médicos, salvo que se produzca un hidrocolpos en los primeros meses de vida. De no presentarse hidrocolpos el tabique sigue silencioso durante la niñez hasta cuando se forme el hematocolpos en la pubertad temprana.

El tabique impide que el material formado por moco de las glándulas cervicales y exudados vaginales fisiológicos salga al exterior; este material es el resultante de la estimulación placentaria hormonal materna pasiva. Si las secreciones son abundantes es lógico que se forme una colección intravaginal llamada hidrocolpos o mucocolpos. Cuando la secreción vaginal es muy escasa no se forma hidrocolpos y entonces la anomalía pasa inadvertida. En la pubertad temprana el tabique se hace siempre sintomático al formarse un hematocolpos causante de criptomenorrea (3) y de pubertad retardada.

Para un buen observador y conocedor de la ginecología pediátrica, la falta de secreción mucosa vaginal en la vulva

* Del Servicio de Ginecología Pediátrica del Hospital Erasmo Meoz. Cúcuta en los primeros días de vida lo lleva a practicar un detenido examen del canal vaginal y a formular un diagnóstico seguro y oportuno.

Dos historias clínicas nos servirán de guía para el presente estudio.

\section{TABIQUE EN RECIEN NACIDAS Material}

Historia clínica 365713. Clínica Infantil del Hospital San Juan de Dios. Cúcuta.

A.M. de cuatro meses de edad, nacida en parto normal después de una gestación de 40 semanas. No hay datos sobre presencia o ausencia de moco vaginal en el período neonatal inmediato. A los dos meses de edad aparece una masa suprapúbica, indolora, de crecimiento progresivo. A los cuatro meses la masa rebasa 7 centímetros por encima del pubis sobre la línea media. Los genitales externos son normales. Himen normal con hendidura himeneal central. $\mathrm{Al}$ investigar la permeabilidad del canal vaginal se observa que está obstruido medio centímetro por encima del himen. A la palpación abdominorectal se aprecia que la masa está por delante de la ampolla rectal y se inicia sobre el sitio de obstrucción. A la vaginoscopia se ve un tabique transversal completo en el tercio inferior de la vagina. A través del tabique y con fines didácticos se inyecta medio centímetro de medio de contraste y se toma una placa radiográfica (figura 1 ). Se encuentra una masa quística de 10 centímetros de largo por 6 de ancho. La urografía excretora es normal. La ecografía muestra un hidrocolpos. Con la ayuda del vaginoscopio, bajo anestesia general, se practica resección circular del tabique y se toman cuatro puntos hemoștáticos. Espontáneamente salen 160 centímetros de material mucoso lechoso aséptico. No se deja 
medicación alguna posterior al procedimiento, ni drenes vaginales, ni se hace limpieza vaginal postoperatoria inmediata. Se informa a la familia la necesidad de practicar un examen ginecológicó en la adolescencia para decidir una conducta definitiva en relación con la base circular del tabique que posiblemente va a producir alguna estrechez en el calibre del canal motivo de dispareunia o de distocia obstétrica.

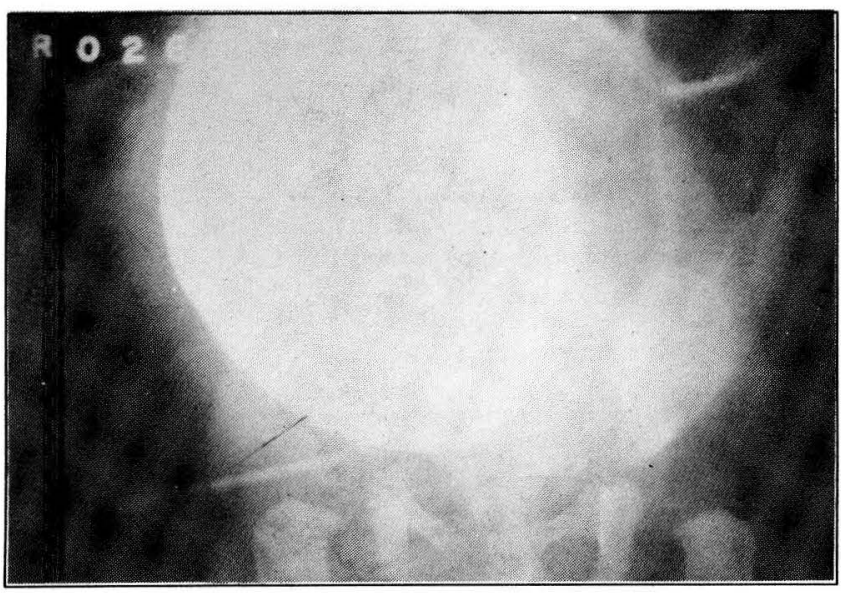

\section{Figura 1}

$\mathrm{Al}$ estudio radiológico se encuentra una masa quística, retropúbica de 10 $\mathrm{cm}$. de largo por 6 de ancho. Se le ha inyectado medio centímetro de medio de contraste. Corresponde a la parte superior de la vagina. Hidrocolpos en una niña de cuatro meses de edad.

\section{Comentarios}

El tabique vaginal transverso se presenta entre uno por 6.000 y uno por 80.000 recién nacidas, según diferentes autores $(5,13)$. Algunos investigadores creen que es una anomalía hereditaria recesiva autosómica $(4,11)$. Su presencia puede ser causa de hidrocolpos también llamado mucocolpos. Su hallazgo es más frecuente que el del himen imperforado.

Godefroy en 1856 publicó la primera descripción que se conoce sobre un caso de hidrocolpos (7). En 1872 Bonnegaze describió su tratamiento (10). Delaunay presentó en 1877 el primer trabajo completo sobre tabique vaginal transverso (6).

El tabique vaginal transverso es una membrana fibromuscular que obstruye total o parcialmente la luz del canal genital. Puede ser total o imperforado y parcial o perforado, en este caso con una ranura central o lateral.

$\mathrm{Su}$ espesor es variable y su calibre por lo general es lenticular. Algunos autores han encontrado varios tabiques dentro de una misma vagina (8); personalmente sólo hemos visto un tabique único.

Puede estar constituido por una lámina celular con fibras musculares y elásticas o por superposición de dos mucosas (8). En la mayoría de los casos se encuentra una superficie interna cubierta por epitelio cilíndrico glandular y una superficie externa o inferior tapizada por epitelio escamoso estratificado y en medio de estas dos capas un tejido colágeno laxo con fibras reticulares elásticas irriga- das por pequeños vasos (12). Desde el trabajo de Kanagasutheran y Dessnayake en 1958 se acepta que estos tabiques se deben a una proliferación anormal, durante la vida embrionaria, del mesodermo que rodea la vagina (9); por un defecto de canalización de la placa vaginal o del tejido que se constituye entre la placa y los ductos mullerianos, el mesodermo circular invade la placa vaginal e impide la perfecta canalización.

El 50\% de los septos transversos localizan en el tercio medio de la vagina, el $20 \%$ en el tercio superior o distal y el $30 \%$ en el tercio proximal o inferior $(1,8)$. Los tabiques del tercio superior casi siempre son incompletos, perforados.

La única manifestación que presenta la recién nacida a término con tabique transverso es la ausencia total de secreción mucosa vaginal al nacer y en período postnatal inmediato y únicamente a éstas es a quienes se les debe investigar la permeabilidad del canal genital. En cambio las recién nacidas prematuras nunca presentan la secreción mucosa vaginal (2) y de ahí la necesidad de investigar a todas ellas la permeabilidad del conducto vaginal.

La falta de secreción vaginal en recién nacidas a término se observa cuando hay cualquier obstrucción del canal vaginal: himen imperforado, septo transverso completo, atresia o agenesia vaginal o cervical y por ello se debe investigar por la clínica y por la ecografía la causa de la obstrucción con miras de poder adelantar el tratamiento adecuado.

En algunas recién nacidas con tabique vaginal transverso se produce abundante secreción, la que no sale a la vulva sino que se acumula dentro de la cavidad vaginal; la vagina se dilata entonces y da como resultado la formación de una masa quística retropúbica, a presión, sobre la línea media, que crece con cierta rapidez y puede producir, según el tamaño, obstrucción del aparato urinario, y aún edema de miembros inferiores por compresión vascular completa: es el hidrocolpos. En este caso, a medida que crece el hidrocolpos, la cúpula vaginal va rechazando hacia la cavidad abdominal tanto al útero como a las trompas. En algunas oportunidades la secreción que llena la vagina pasa al útero formando el hematometra; sigue luego hacia las trompas ocasionando el hematosálpinx y se vacía por último a la cavidad pélvica produciendo entonces peritonitis fibrosa, calcificaciones peritoneales y obstrucción intestinal $(5,14)$.

El diagnóstico diferencial del hidrocolpos se debe hacer con retención urinaria, quistes del uraco, teratoma presacro, meningocele presacro, duplicación del rectosigmoide, neoplasias ováricas y dilatación superior de los ureteres, a más de ciertas alteraciones del colon como enfermedad de Hirschsprung.

El diagnóstico del septo transverso se confirma con la vaginoscopia y el del hidrocolpos con la ecografía y con la radiografía abdominal.

El tabique vaginal transverso va acompañado con frecuencia por otras malformaciones como anomalías del aparato urinario en el $20 \%$ de los casos: hipoplasia renal, ptosis renal, duplicación ureteral, fístula vesicovaginal, agenesia renal unilateral. Se puede encontrar también alteraciones anorrectales como imperforación anal, persistencia de la cloaca y fístula rectovaginal e incluso malformaciones cardíacas como coartación de la aorta y defectos del septo atrial. También se ha descrito polidactilia y defectos del septo ventricular. Ultimamente se ha encontra- 
do asociado a útero didelfo y a doble vagina, y se ha descrito el Síndrome de McKusick-Faufman que consiste en septo vaginal transverso completo más atresia de vagina, hidrometrocolpos, duplicación útero vaginal, anomalías anorrectales, polidactilia postaxial y lesiones cardíacas (4).

\section{EN LA PUBERTAD \\ Material}

\section{H.C. 470985. Hospital Erasmo Meoz. Cúcuta.}

Paciente de 15 años que presenta telarca a los 12 años y puberquia a los $12^{1 / 2}$. Consulta por amenorrea primaria y dolor retropúbico desde la edad de 13 años, que se presenta cada mes y siempre con mayor intensidad. Desarrollo de los caracteres sexuales secundarios un poco retardados; genitales externos normales. No hay datos de examen de la recién nacida. Himen desgarrado, vida sexual activa. Dispareunia. A la especuloscopia se observa un tabique vaginal transverso completo en el tercio superior de la vagina. Al tacto vaginal se encuentra una vagina corta de 5 centímetros y una masa de forma irregular que ocupa la parte superior de la vagina y el útero. A la ecografía se observa hematocolpos, hematometra y hematosálpinx. Como el sitio de implantación del tabique es reducido, estrecho por constricción anular, se emplea la vía abdomino perineal para el acto quirúrgico. Se encuentra abundante cantidad de sangre en el fondo de Douglas. Se extirpa el tabique y se deja que la sangre retenida drene por vía vaginal espontáneamente. Ocho días más tarde se hace la plastia de vagina para normalizar la luz del canal en el sitio de la estrechez. Se advierte a la paciente la posibilidad de la aparición posterior de una endometriosis y de problemas de esterilidad de origen tubario.

\section{Comentarios}

El tabique vaginal transverso puede permanecer asintomático durante todo el período neonatal y la infancia, pero irremediablemente se hace sintomático cuando llega la pubertad.

Al presentarse la menarca, el sangrado menstrual queda retenido en la vagina, aumenta el volumen del líquido que en ella puede haber y da inicio al hematocolpos. Con la aparición de cada nueva menstruación se incrementa el volumen del hematocolpos y se presenta dolor cada vez más intenso y aumento de la masa endovaginal (8). La adolescente tiene entonces un hematocolpos, el que de no ser tratado de inmediato forma posteriormente el hematometra, luego el hematosálpinx y por último el escurrimiento de sangre menstrual a la cavidad peritoneal, posible causa de endometriosis.

El tabique vaginal transverso completo es la causa más frecuente de criptomenorrea (7) lo mismo que de pubertad retardada de origen anatómico (15-17).

En las adolescentes el diagnóstico del tabique se hace por vaginoscopia o por especuloscopia según el estado del himen. El hematocolpos, el hematometra y el hematosálpinx se diagnostican por tacto rectal o vaginal y por ecografía.

Ultimamente se acepta que procesos inflamatorios infecciosos, traumáticos o cuerpos extraños pueden ser causantes de formación de septos transversos de comienzo postnatal (5).

\section{Tratamiento}

En la mayoría de los casos el tratamiento tanto del mucocolpos como del hematocolpos es extremadamente sencillo; perforar el septo por vía vaginal, abrir un ojal con un bisturí en bayoneta y dejar que la masa quística drene espontáneamente. Se debe tener cuidado en no lesionar vejiga ni recto.

Entre mayor sea la distensión quística, mayor la facilidad para la pequeña cirugía y menor el riesgo de lesionar órganos vecinos.

Cuando el tabique está ubicado en la parte superior de la vagina, y ésta se encuentra muy distendida es necesario practicar una laparotomía: se abre la vagina por el vértice de su porción dilatada; con un dilatador de Hegar No. 6 introducido por la vagina se identifica y se pone tensa la membrana obstructiva y por este sitio se abre el ojal al tabique. Se sutura luego la vagina; la evacuación del material retenido se hace espontáneamente por vía vaginal.

El aspecto más importante del hidrocolpos y del hematocolpos es el diagnóstico con miras a practicar un tratamiento adecuado y evitar la ejecución de laparotomías innecesarias y aún de histerectomías a esta edad.

Algo importante es no introducir torundas ni mechas dentro de la cavidad quística por el peligro de instaurar una infección postquirúrgica, como también esperar con paciencia que se evacúe lentamente la totalidad de las secreciones retenidas.

\section{BIBLIOGRAFIA}

1. RockJ., Schlaff W. The obstetric consequences of uterovaginal anomalies. Fertil Steril 1985; 43: 681.

2. Duarte-Contreras A. Valor semiológico de la secreción mucosa genital en la recién nacida. En prensa. Rev. Col. Obstet. Ginecol. 1993.

3. Duarte-Contreras A. Criptomenorrea. Rev. Col. Obstet. Gynecol. 1989; 40: 313-318.

4. Meinhard R., Shaw A. The McKusick-Kaufman syndrome: Recessively inherited vaginal atresia, hydrometrocolpos, utero vaginal duplications, anorectal anomalies, postaxial polydactyly, and congenital hear disease. J. Pediatr. 1979; 94: 776.

5. Sweet CR., Galle PC., McRae MA., Denby JL. Transverse vaginal septum: A diagnosis at tree months of age. Adolesc. Ped. Gynecol. 1991; 4: 35-38.
6. Delaunay JV. Etude sur le cloisonnement transversal du vagin complet et incomplet, d'origine congenital. Thèse Paris 1877; 46: 130.

7. Godefroy M. Imperforation de la membrane hymen. Louccette Franc. Gaz d'Hosp. 1856; 29: 567.

8. Granjon A. Malformations congenitales. Cloissonnements du vagin. Encyclopedie médco-chirurgicale Gynecologie. 1952; 120 A 20-1.

9. Kanagasuntheran R., Dassanayake A. Nature of the obstructing membrane in primary cryptomenorrhea. J. Obstet. and Gynaec. Brit. Emp. 1958; 65: 487.

10. Bonnecaze. Traitement chirurgical des imperfections du vagin. Thèse Paris. 1872. 
11. McKusick VA., Bauer RL. Koop CE y cols. Hydrometrocolpos as a simple inherited malformation. JAMA 1964; 189: 813-816.

12. Blair Bell W. Further investigations into the chemical composition of menstrual fluid and the secretions of the vagina. J. Obstet. and Gynaec. Brit. Emp. 1912; 21: 209.

13. Rock JA., Azzis R. Anomalías genitales en la infancia. Clínicas Obstet. y Ginecol. 1987; 3: 647-661.

14. Dewhurst SJ. Malformations of the genital tract. Hydrocolpos. En: Practical pediatric and adolescent gynecology. Marcel Dekker Inc. Ed. $1980 ; 62$.
15. Dewhurst SJ. Abnormalities presenting in the pubertal patient. Hematocolpos. En: Practical pediatric and adolescent gynecology. Marcel Dekker Inc. Ed. 1980; 67.

16. Dewhurst SJ. Delayed Puberty: Haematocolpos. En: Female puberty and its abnormalities. Churchill Livingstone. Ed. Edimburgh. 1984; 127.

17. Duarte-Contreras A. Malformaciones no genéticas de los órganos genitales. Vagina tabicada. En: Ginecología de la niña y de la adolescente. Salvat Editores Colombiana S.A. 1988; 66.

\section{EVENTOS}

XVI CONGRESO NACIONAL DE CIRUGIA 7 al 10 de noviembre de 1993 Centro de Convenciones Gonzalo Jin.énez de Quesada, Informes: Colegio Colombiano de Cirujanos Tel. 6169268

\section{CONGRESO LATINOAMERICANO DE OBSTETRICIA Y GINECOLOGIA}

XIX CONGRESO CENTROAMERICANO DE OBSTETRICIA Y GINECOLOGIA FLASOG 93 Noviembre 28 a Diciembre 3 - Centro de Convenciones Atlapa - Panamá, Informes Federación Colombiana de Sociedades de Obstetricia y Ginecología. Tels. 2681485 - 2444692 Ext. 22.

XIX CONGRESO NACIONAL DE OBSTETRICIA Y GINECOLOGIA, Marzo 2 al 5 de 1994 Centro de Convenciones Gonzalo Jiménez de Quesada, Informes Sociedad Colombiana de Obstetricia y Ginecología. Tels. 2444419 y 2681485.

CONGRESO MUNDIAL DE ENDOSCOPIA GINECOLOGICA del 2 al 5 de diciembre de 1993 en Bombay, India. Informes Telfaz Nó. 91-22-3871415 Tel. 351522, 3885127 en Bombay.

IV CONGRESO MUNDIAL DE ENDOMETRIOSIS del 25 al 28 de mayo de 1994 en Salvador Baha Brazil. Informes Secretaría Congreso Rua Caetano Moura, 35 - Federacao, 40210-431 - Salvador Tel. (55-71) 247-0350. 